\title{
Pacific
}

Journal of

Mathematics

\section{MLUR RENORMINGS OF BANACH SPACES}

Patrick Dowling, Zhibao Hu and Mark ANDREw SMith 


\title{
MLUR RENORMINGS OF BANACH SPACES
}

\author{
Patrick N. Dowling, Zhibao Hu and Mark A. Smith
}

A specific construction is given on a Banach space $X$ with a 1-unconditional basis which yields an equivalent norm on $X$ that is midpoint locally uniformly rotund. This construction, when applied to $\ell^{2}$, confirms a suspicion of K.W. Anderson and answers a question of R.E. Megginson; when applied to an example of $M$. Talagrand, it improves an example of P-K. Lin.

\section{Introduction.}

In 1960, K.W. Anderson [A] systematically studied the geometrical notion of midpoint local uniform rotundity in Banach spaces (definitions are given in the next section), a notion which had been known earlier to G. Lumer and M.M. Day. His motivation for such a study was to find the correct rotundity notion in a reflexive Banach space $X$ that would be in duality to the smoothness notion of Fréchet differentiability of the norm in the conjugate space $X^{*}$. Although this hoped-for duality did not materialize, Anderson did discover that the combination of strict convexity and the Kadec-Klee property in reflexive Banach spaces is in full duality with Fréchet differentiability of the conjugate norm; he also showed that this combination in a reflexive Banach space implies midpoint local uniform rotundity. Anderson strongly suspected that the last mentioned implication could not be reversed (that is, that, in reflexive Banach spaces, midpoint local uniform rotundity is not equivalent to the combination of strict convexity and the Kadec-Klee property). However, he was unable to provide an example; in fact, he left open the question of whether midpoint local uniform rotundity implies the Kadec-Klee property in any Banach space.

In 1981, M.A. Smith [S] gave an example of a midpoint locally uniformly rotund Banach space that fails to have the Kadec-Klee property, which thus answered Anderson's question in the negative. But the example that Smith gave is a renorming of $c_{0}$ (and hence a nonreflexive space), thereby leaving Anderson's suspicion still unconfirmed.

In 1983, R.E. Megginson [M], during his investigation of the semi-KadecKlee condition and nearest-point properties in Banach spaces, posed the question: Are the reflexive midpoint locally uniformly rotund spaces exactly 
the strongly rotund Banach spaces? This question, as Megginson points out, is equivalent to Anderson's question of whether reflexive midpoint locally uniformly rotund spaces have the Kadec-Klee property. Megginson gave a third equivalent formulation of this question in terms of distance minimizing sequences in a closed ball for a point, a formulation that led him to join Anderson in the strong suspicion that the answer must be negative; but still no example was known.

Backtracking chronologically, in 1980, M.A. Smith and B. Turett [S-T], during their examination of which generalizations of uniform rotundity lift from a Banach space $X$ to the Lebesgue-Bochner function space $L^{p}(\mu, X)$, posed the question: If $X$ is strictly convex and has the Kadec-Klee property and if $1<p<\infty$, then does the space $L^{p}(\mu, X)$ have the Kadec-Klee property? They noted that the answer is affirmative, whenever $X$ is reflexive; they also showed that without strict convexity the Kadec-Klee property need not lift from $X$ to $L^{p}(\mu, X)$, even when $X$ is reflexive.

In 1987, P-K. Lin [L] constructed an example of a strictly convex Banach space $X$ with the Kadec-Klee property such that $L^{p}(\mu, X)$ fails to have the Kadec-Klee property, thus providing a negative answer to the question of Smith and Turett. This space $X$ is not midpoint locally uniformly rotund.

In this paper, a specific construction is given on a Banach space $X$ with a 1-unconditional basis which yields an equivalent norm on $X$ that is midpoint locally uniformly rotund. This construction, when applied to $\ell^{2}$, produces the example sought after by both Anderson and Megginson and finally confirms their suspicions that a reflexive midpoint locally uniformly rotund Banach space need not have the Kadec-Klee property. This application to $\ell^{2}$ also improves the example of Smith cited above by providing a reflexive space with the specified properties. An application of the construction to an example of $\mathrm{M}$. Talagrand [T] produces a midpoint locally uniformly rotund Banach space $X$ with the Kadec-Klee property such that $L^{p}(\mu, X)$ fails to have the Kadec-Klee property, thereby improving the example of Lin cited above by upgrading the level of rotundity of both $X$ and $L^{p}(\mu, X)$ from strict convexity to midpoint local uniform rotundity. In addition, the space $X$ produced by this second application has the Radon-Nikodým property and the Schur property; yet the space $h^{p}(D, X)$ of vector-valued harmonic functions fails to have the Kadec-Klee property with respect to uniform convergence on compact subsets of the unit disc $D$; this example complements some recent work of the authors [D-H-S]. 


\section{Definitions and Preliminaries.}

Throughout this paper $X$ will denote a real Banach space, $B_{X}$ will denote the closed unit ball of $X$ and $S_{X}$ will denote the unit sphere of $X$. A sequence $\left\{e_{n}\right\}_{n=0}^{\infty}$ in $X$ is called a 1-unconditional basis for $X$ provided $\left\{e_{n}\right\}_{n=0}^{\infty}$ is an unconditional basis for $X$ with unconditional constant 1 ; that is, for every choice of scalars $\left\{x^{n}\right\}_{n=0}^{\infty}$ such that $\sum_{n=0}^{\infty} x^{n} e_{n}$ converges and for every choice of bounded scalars $\left\{\lambda_{n}\right\}_{n=0}^{\infty}$, it follows that

$$
\left\|\sum_{n=0}^{\infty} \lambda_{n} x^{n} e_{n}\right\| \leq \sup _{n}\left|\lambda_{n}\right|\left\|\sum_{n=0}^{\infty} x^{n} e_{n}\right\| .
$$

Recall that a Banach space with an unconditional basis can be renormed to have a 1-unconditional normalized basis; see [L-T, pp. 18-19].

For a given measure space $(\Omega, \Sigma, \mu)$ and $1<p<\infty$, the symbol $L^{p}(\mu, X)$ will denote the usual Lebesgue-Bochner function space $L^{p}(\Omega, \Sigma, \mu, X)$; see [D-U, p. 97]. For $1<p<\infty$, the symbol $h^{p}(D, X)$ will denote the space of $X$-valued harmonic functions defined on the open unit disc $D$ in the complex plane which have finite p-norm; see [D-H-S]. A sequence $\left\{f_{n}\right\}_{n=1}^{\infty}$ in $h^{p}(D, X)$ is said to converge with respect to $\beta$ to $f$ in $h^{p}(D, X)$ provided

$$
\lim _{n \rightarrow \infty}\left(\sup \left\{\left\|f_{n}(z)-f(z)\right\|: z \in K\right\}\right)=0
$$

for all compact subsets $K$ of $D$.

A Banach space $X$ is said to have the Kadec - Klee property (KK) provided that whenever $\left\{x_{n}\right\}_{n=1}^{\infty}$ is a sequence in $S_{X}$ and $x$ is in $S_{X}$ with $x_{n} \rightarrow x$ weakly, it follows that $x_{n} \rightarrow x$ in norm. For $1<p<\infty$ and $X$, the space $h^{p}(D, X)$ is said to have the Kadec - Klee property with respect to $\beta$ convergence $(\mathrm{KK}(\beta))$ provided that whenever $\left\{f_{n}\right\}_{n=1}^{\infty}$ is a sequence in $S_{h^{p}(D, X)}$ and $f$ is in $S_{h^{p}(D, X)}$ with $f_{n} \rightarrow f$ with respect to $\beta$, it follows that $f_{n} \rightarrow f$ in norm.

The following is a well-known list of geometrical notions in a Banach space; the list is given in the order of increasing strength.

(i) A point $x$ in $B_{X}$ is called an extreme point of $B_{X}$ provided $x$ is not the midpoint of any non-trivial line segment lying in $B_{X}$. A Banach space $X$ is said to be strictly convex provided every $x$ in $S_{X}$ is an extreme point of $B_{X}$.

(ii) A point $x$ in $B_{X}$ is called a strongly extreme point of $B_{X}$ provided that whenever $\left\{x_{n}\right\}_{n=1}^{\infty}$ is a sequence in $X$ with $\left\|x \pm x_{n}\right\| \rightarrow 1$, it follows that $x_{n} \rightarrow 0$ in norm. A Banach space $X$ is said to be midpoint locally uniformly rotund (MLUR) provided every $x$ in $S_{X}$ is a strongly extreme point of $B_{X}$. 
(iii) A point $x$ in $B_{X}$ is called a denting point of $B_{X}$ provided $x$ is not an element of the closed convex hull of $\left\{y \in B_{X}:\|y-x\|>\varepsilon\right\}$ for each $\varepsilon>0$. A Banach space $X$ is said to have property $(G)$ provided every $x$ in $S_{X}$ is a denting point of $B_{X}$.

It should also be noted that, in any Banach space, property $(\mathrm{G})$ implies $\mathrm{KK}$ and, in a Banach space that contains no isomorphic copy of $\ell^{1}$, the combination of $\mathrm{KK}$ and strict convexity implies property $(\mathrm{G})$; see [L-L1].

\section{The Construction.}

Let $X$ be a Banach space with a 1-unconditional normalized basis $\left\{e_{j}\right\}_{j=0}^{\infty}$. For $n \geq 0$ and $x=\sum_{j=0}^{\infty} x^{j} e_{j}$, define $\beta_{n}: X \rightarrow \mathbb{R}$ by

$$
\beta_{n}\left(\sum_{j=0}^{\infty} x^{j} e_{j}\right)=\max \left\{\left|x^{0}\right|,\left|x^{n}\right|,\left\|\sum_{j=n+1}^{\infty} x^{j} e_{j}\right\|\right\} .
$$

It is easy to show that each $\beta_{n}$ is a lattice seminorm on $X$ and that $\beta_{n}(x) \leq$ $\|x\| \leq 2 \beta_{0}(x)$ for all $x$ in $X$. Now, for each $n \geq 0$, define $\sigma_{n}: X \rightarrow \mathbb{R}$ by

$$
\sigma_{n}\left(\sum_{j=0}^{\infty} x^{j} e_{j}\right)=\beta_{n}\left(\sum_{j=0}^{\infty}\left(x^{j}\right)^{+} e_{j}\right)+\beta_{n}\left(\sum_{j=0}^{\infty}\left(x^{j}\right)^{-} e_{j}\right) .
$$

It is straightforward to show that each $\sigma_{n}$ is a seminorm on $X$ and, for each $n \geq 0$ and each $x$ in $X$, that $\beta_{n}(x) \leq \sigma_{n}(x) \leq 2 \beta_{n}(x)$ and so $\sigma_{n}(x) \leq 2\|x\|$. Define $T_{n, m}: X \rightarrow X$ by

$$
T_{0, m}\left(\sum_{j=0}^{\infty} x^{j} e_{j}\right)=x^{0} e_{0}+\sum_{j=m}^{\infty} x^{j} e_{j} \quad \text { for } m>0
$$

and

$$
T_{n, m}\left(\sum_{j=0}^{\infty} x^{j} e_{j}\right)=x^{0} e_{0}+x^{n} e_{n}+\sum_{j=m}^{\infty} x^{j} e_{j} \quad \text { for } m>n>0
$$

It is clear that each $T_{n, m}$ is linear and that $\left\|T_{n, m}(x)\right\| \leq\|x\|$ for each $x$ in $X$, since $\left\{e_{j}\right\}_{j=0}^{\infty}$ is a 1-unconditional basis. Now, for $n \geq 1$, choose $\alpha_{n}>0$ and, for $m>n \geq 0$, choose $\alpha_{n, m}>0$ such that

$$
\sum_{n \geq 1} \alpha_{n}^{2}=\sum_{m>n \geq 0} \alpha_{n, m}^{2}=1
$$

Define $T: X \rightarrow \ell^{2}$ by

$$
T\left(\sum_{j=0}^{\infty} x^{j} e_{j}\right)=\left(x^{0}, \alpha_{1} x^{1}, \alpha_{2} x^{2}, \ldots\right)
$$


Then $T$ is linear and $\|T(x)\|_{2} \leq 2\|x\|$ for all $x$ in $X$. Finally, define the following norm on $X$ : for $x$ in $X$, let

$(*)$

$$
\begin{aligned}
\|x\|_{\mathrm{M}}=\left(\beta_{0}(x)^{2}+\sum_{n \geq 1} \alpha_{n}^{2} \beta_{n}(x)^{2}\right. & \\
& \left.\quad+\sum_{m>n \geq 0} \alpha_{n, m}^{2} \sigma_{n}\left(T_{n, m}(x)\right)^{2}+\|T(x)\|_{2}^{2}\right)^{\frac{1}{2}} .
\end{aligned}
$$

Then

$$
\begin{aligned}
\frac{1}{2}\|x\| & \leq \beta_{0}(x) \\
& \leq\|x\|_{\mathrm{M}} \\
& \leq\left(\|x\|^{2}+\sum_{n \geq 1} \alpha_{n}^{2}\|x\|^{2}+\sum_{m>n \geq 0} \alpha_{n, m}^{2}(2\|x\|)^{2}+(2\|x\|)^{2}\right)^{\frac{1}{2}} \\
& =\sqrt{10}\|x\|
\end{aligned}
$$

and hence $\|\cdot\|_{M}$ is an equivalent norm on $X$.

Theorem. Let $X$ be a Banach space with a 1-unconditional basis $\left\{e_{n}\right\}_{n=0}^{\infty}$ and let $\|\cdot\|_{M}$ be the equivalent norm on $X$ defined by $(*)$. Then the Banach space $\left(X,\|\cdot\|_{M}\right)$ is MLUR and $\lim _{n \rightarrow \infty}\left\|e_{0}+e_{n}\right\|_{M}=\left\|e_{0}\right\|_{M}=2$.

Proof. It is a straightforward computation to see that $\lim _{n \rightarrow \infty}\left\|e_{0}+e_{n}\right\|_{\mathrm{M}}$ $=\left\|e_{0}\right\|_{\mathrm{M}}=2$ and so it remains to show only that $\left(X,\|\cdot\|_{\mathrm{M}}\right)$ is MLUR. Toward this end, suppose $x$ is in $X$ with $\|x\|_{M}=1$ and $\left\{x_{k}\right\}_{k=1}^{\infty}$ is a sequence in $X$ with $\left\|x \pm x_{k}\right\|_{M} \rightarrow 1$. Then the definition of $\|\cdot\|_{M}$ yields the following three equations:

$$
\begin{gathered}
\lim _{k \rightarrow \infty} \beta_{n}\left(x \pm x_{k}\right)=\beta_{n}(x) \quad \text { for all } n \geq 0, \\
\lim _{k \rightarrow \infty} \sigma_{n}\left(T_{n, m}\left(x \pm x_{k}\right)\right)=\sigma_{n}\left(T_{n, m}(x)\right) \quad \text { for all } m>n \geq 0
\end{gathered}
$$

and

$$
\lim _{k \rightarrow \infty}\left\|T\left(x \pm x_{k}\right)\right\|_{2}=\|T(x)\|_{2} ;
$$

these equations follow by first observing that

$$
2\left(\left\|x+x_{k}\right\|_{M}^{2}+\left\|x-x_{k}\right\|_{M}^{2}\right)-\|2 x\|_{M}^{2} \rightarrow 0,
$$

by then rewriting this using $(*)$ and finally by grouping "like" terms together and applying the Lemma in [S] (see [S, p. 278] for details). Since $\left(\ell^{2},\|\cdot\|_{2}\right)$ is MLUR, equation (3) yields that $\lim _{k \rightarrow \infty}\left\|T\left(x_{k}\right)\right\|_{2}=0$ and hence

$$
\lim _{k \rightarrow \infty} x_{k}^{j}=0 \quad \text { for all } j \geq 0, \text { where } x_{k}=\sum_{j=0}^{\infty} x_{k}^{j} e_{j} .
$$


Since $x$ is not zero, there exists $n_{0}$ in $\mathbb{N} \cup\{0\}$ such that $x^{n_{0}} \neq 0$ and $x^{j}=0$ for each $j<n_{0}$. Without loss of generality, assume $x^{n_{0}}>0$. Invoking (4), it can also be assumed that $x_{k}^{j}=0$ for $0 \leq j \leq n_{0}$ and for all $k$ in $\mathbb{N}$. To show $\left(X,\|\cdot\|_{\mathrm{M}}\right)$ is MLUR, it must be shown that $x_{k} \rightarrow 0$ in norm. Suppose this is not the case. Then, by passing to a subsequence if necessary, there exists $\varepsilon>0$ such that $\left\|x_{k}\right\| \geq \varepsilon$ for all $k$ in $\mathbb{N}$. There exists $m>n_{0}$ such that $\left\|\sum_{j=m}^{\infty} x^{j} e_{j}\right\|<\frac{1}{16} \varepsilon$ and hence

$$
\begin{aligned}
\left|\sigma_{n_{0}}\left(T_{n_{0}, m}(x)\right)-x^{n_{0}}\right| & =\left|\sigma_{n_{0}}\left(T_{n_{0}, m}(x)\right)-\sigma_{n_{0}}\left(T_{n_{0}, m}\left(x^{n_{0}} e_{n_{0}}\right)\right)\right| \\
& \leq \sigma_{n_{0}}\left(T_{n_{0}, m}\left(x-x^{n_{0}} e_{n_{0}}\right)\right) \\
& =\sigma_{n_{0}}\left(\sum_{j=m}^{\infty} x^{j} e_{j}\right) \\
& \leq 2\left\|\sum_{j=m}^{\infty} x^{j} e_{j}\right\| \\
& <\frac{\varepsilon}{8} .
\end{aligned}
$$

Now, $\lim _{k \rightarrow \infty} \sigma_{n_{0}}\left(T_{n_{0}, m}\left(x \pm x_{k}\right)\right)=\sigma_{n_{0}}\left(T_{n_{0}, m}(x)\right)$, by (2), and so there exists $k_{0}$ in $\mathbb{N}$ such that, for all $k \geq k_{0}$,

$$
\left|\sigma_{n_{0}}\left(T_{n_{0}, m}\left(x \pm x_{k}\right)\right)-\sigma_{n_{0}}\left(T_{n_{0}, m}(x)\right)\right|<\frac{\varepsilon}{8} .
$$

By (4), there exists $k_{1} \geq k_{0}$ such that $\left\|\sum_{j=0}^{m-1} x_{k}^{j} e_{j}\right\|<\frac{1}{8} \varepsilon$ for all $k \geq k_{1}$. Now, for all $k \geq k_{1}$,

$$
\left|\sigma_{n_{0}}\left(T_{n_{0}, m}\left(x \pm x_{k}\right)\right)-\sigma_{n_{0}}\left(T_{n_{0}, m}\left(x^{n_{0}} e_{n_{0}} \pm x_{k}\right)\right)\right| \leq \sigma_{n_{0}}\left(T_{n_{0}, m}\left(x-x^{n_{0}} e_{n_{0}}\right)\right)<\frac{\varepsilon}{8}
$$

and hence

$$
\begin{aligned}
& \mid \sigma_{n_{0}}\left(T _ { n _ { 0 } , m } \left(x^{n_{0}} e_{n_{0}}\right.\right.\left.\left.+x_{k}\right)\right)-x^{n_{0}} \mid \\
& \leq \mid \sigma_{n_{0}}(\left.T_{n_{0}, m}\left(x^{n_{0}} e_{n_{0}}+x_{k}\right)\right)-\sigma_{n_{0}}\left(T_{n_{0}, m}\left(x+x_{k}\right)\right) \mid \\
&+\left|\sigma_{n_{0}}\left(T_{n_{0}, m}\left(x+x_{k}\right)\right)-\sigma_{n_{0}}\left(T_{n_{0}, m}(x)\right)\right| \\
&+\left|\sigma_{n_{0}}\left(T_{n_{0}, m}(x)\right)-x^{n_{0}}\right| \\
&<\frac{3 \varepsilon}{8}
\end{aligned}
$$

Similarly, it can be shown that, for all $k \geq k_{1}$,

$$
\left|\sigma_{n_{0}}\left(T_{n_{0}, m}\left(x^{n_{0}} e_{n_{0}}-x_{k}\right)\right)-x^{n_{0}}\right|<\frac{3 \varepsilon}{8} .
$$


Note that, for all $k \geq k_{1}$,

$$
\begin{aligned}
\sigma_{n_{0}}\left(T_{n_{0}, m}\left(x^{n_{0}} e_{n_{0}}+x_{k}\right)\right) & =\beta_{n_{0}}\left(x^{n_{0}} e_{n_{0}}+\sum_{j=m}^{\infty}\left(x_{k}^{j}\right)^{+} e_{j}\right)+\beta_{n_{0}}\left(\sum_{j=m}^{\infty}\left(x_{k}^{j}\right)^{-} e_{j}\right) \\
& \geq x^{n_{0}}+\beta_{n_{0}}\left(\sum_{j=m}^{\infty}\left(x_{k}^{j}\right)^{-} e_{j}\right)
\end{aligned}
$$

and thus $\beta_{n_{0}}\left(\sum_{j=m}^{\infty}\left(x_{k}^{j}\right)^{-} e_{j}\right)<\frac{3}{8} \varepsilon$. Similarly, it follows that

$$
\beta_{n_{0}}\left(\sum_{j=m}^{\infty}\left(-x_{k}^{j}\right)^{-} e_{j}\right)<\frac{3}{8} \varepsilon
$$

that is, $\beta_{n_{0}}\left(\sum_{j=m}^{\infty}\left(x_{k}^{j}\right)^{+} e_{j}\right)<\frac{3}{8} \varepsilon$ for all $k \geq k_{1}$. Finally, for all $k \geq k_{1}$, it now follows that

$$
\begin{aligned}
\left\|x_{k}\right\| & \leq\left\|\sum_{j=0}^{m-1} x_{k}^{j} e_{j}\right\|+\left\|\sum_{j=m}^{\infty} x_{k}^{j} e_{j}\right\| \\
& \leq\left\|\sum_{j=0}^{m-1} x_{k}^{j} e_{j}\right\|+\left\|\sum_{j=m}^{\infty}\left(x_{k}^{j}\right)^{+} e_{j}\right\|+\left\|\sum_{j=m}^{\infty}\left(x_{k}^{j}\right)^{-} e_{j}\right\| \\
& <\frac{\varepsilon}{8}+\frac{3 \varepsilon}{8}+\frac{3 \varepsilon}{8} \\
& =\frac{7 \varepsilon}{8} .
\end{aligned}
$$

This contradicts the fact that $\left\|x_{k}\right\| \geq \varepsilon$ for all $k$ in $\mathbb{N}$. This shows that $\left(X,\|\cdot\|_{\mathrm{M}}\right)$ is MLUR and the proof is complete.

Corollary. Let $X$ be a Banach space with a weakly null 1-unconditional normalized basis $\left\{e_{n}\right\}_{n=0}^{\infty}$ and let $\|\cdot\|_{M}$ be the equivalent norm on $X$ defined by $(*)$. Then $\left(X,\|\cdot\|_{M}\right)$ is MLUR but fails to have $K K$.

Proof. The Banach space $\left(X,\|\cdot\|_{M}\right)$ is MLUR by the Theorem. Since $\left\{e_{n}\right\}_{n=0}^{\infty}$ is weakly null, $\frac{1}{2}\left(e_{0}+e_{n}\right) \rightarrow \frac{1}{2} e_{0}$ weakly and, by the Theorem, $\lim _{n \rightarrow \infty}\left\|\frac{1}{2}\left(e_{0}+e_{n}\right)\right\|_{\mathrm{M}}=\left\|\frac{1}{2} e_{0}\right\|_{\mathrm{M}}=1$. Therefore $\left(X,\|\cdot\|_{\mathrm{M}}\right)$ fails to have $\mathrm{KK}$ and the proof is complete.

\section{Applications.}

In this final section, the two examples advertised in the introduction will be produced by applying the results from the previous section. 
Example 1. A direct application of the Corollary shows that $c_{0}$ and $\ell^{p}$, for $1<p<\infty$, admit equivalent renormings so as to be MLUR and fail to have KK. In particular, there exists a reflexive Banach space that is MLUR but fails to have KK; this is the first known example of a reflexive Banach space in which all closed balls, but not all nonempty closed convex sets, are approximatively compact Chebyshev sets; see [M, Theorem 2.7 and Theorem B.8].

The next goal is to produce an example of a Banach space $X$ which has KK and is MLUR but such that $L^{p}(\mu, X)$ fails to have KK for each $p$ with $1<p<\infty$ and an appropriate measure space $(\Omega, \Sigma, \mu)$. Following the lead of Lin $[\mathbf{L}]$, the example constructed here is also a renorming of Talagrand space $[\mathbf{T}]$.

To begin, recall the following facts about Talagrand space. Let $\Delta=$ $\cup_{n \geq 1}\{0,1\}^{n}$ be the usual dyadic tree. If $\varphi=\left\{\varepsilon_{i}\right\}_{i=1}^{n}$ is in $\{0,1\}^{n}$, write $|\varphi|=n$ and say that $\varphi$ is a node of order $n$. If $\varphi$ and $\psi$ are two nodes, write $\psi \geq \varphi$ whenever $\psi$ extends $\varphi$. Then Talagrand space, $E$, is defined to be the completion of the space of all finitely non-zero functions $e: \Delta \rightarrow \mathbb{R}$ under the norm

$$
\|e\|=\sup _{n \geq 1}\left(\sum_{|\varphi|=n} \sup _{\psi \geq \varphi} e(\psi)^{2}\right)^{\frac{1}{2}} .
$$

Enumerate $\Delta$ as $\left\{\psi_{n}\right\}_{n=1}^{\infty}$ and let $e_{\psi_{n}}: \Delta \rightarrow \mathbb{R}$ be the function that is 1 at $\psi_{n}$ and 0 elsewhere. Then $\left\{e_{\psi_{n}}\right\}_{n=1}^{\infty}$ is a 1-unconditional basis for $E$. Talagrand [T] showed that $E^{*}$ is separable and so $\left\{e_{\psi_{n}}\right\}_{n=1}^{\infty}$ is a shrinking basis for $E$. Hence the sequence of coefficient functionals $\left\{e_{\psi_{n}}^{*}\right\}_{n=1}^{\infty}$ is a 1-unconditional basis for $E^{*}$.

Example 2. Let $X=\mathbb{R} \oplus E^{*}$ with norm, $\|\cdot\|$, given by

$$
\left\|\left(r, e^{*}\right)\right\|=\max \left\{|r|,\left\|e^{*}\right\|\right\}
$$

for $\left(r, e^{*}\right)$ in $\mathbb{R} \oplus E^{*}$. Let $e_{0}=(1,0)$ and $e_{n}=\left(0, e_{\psi_{n}}^{*}\right)$ for $n \geq 1$. Since $E^{*}$ embeds isometrically into $X$, it is easy to check that $\left\{e_{n}\right\}_{n=0}^{\infty}$ is a 1 unconditional basis for $X$ and $\left\|e_{n}\right\|=1$ for all $n \geq 0$. Let $\|\cdot\|_{\mathrm{M}}$ be the MLUR norm on $X$ induced from $\left\{e_{n}\right\}_{n=0}^{\infty}$ via the Theorem. So

$$
\lim _{n \rightarrow \infty}\left\|e_{0}+e_{n}\right\|_{\mathrm{M}}=\left\|e_{0}\right\|_{\mathrm{M}}=2 .
$$

Let $\Omega=\Pi_{n \geq 1}\{0,1\}^{n}$, let $\Sigma$ be the Borel $\sigma$-algebra of subsets of $\Omega$ and let $\mu$ denote the normalized Haar measure on $\Omega$. For each $n$ in $\mathbb{N}$, let $p_{n}$ be the canonical projection from $\Omega$ onto $\{0,1\}^{n}$ and define $g_{n}: \Omega \rightarrow X$ by $g_{n}(\omega)=\frac{1}{2}\left(0, e_{p_{n}(\omega)}^{*}\right)$ for all $\omega$ in $\Omega$. As noted in $[\mathbf{L}]$, the sequence $\left\{g_{n}\right\}_{n=1}^{\infty}$ converges weakly to zero in $L^{p}(\mu, X)$ for each $1<p<\infty$. Define $h: \Omega \rightarrow X$ by $h(\omega)=\frac{1}{2} e_{0}$ for all $\omega$ in $\Omega$. Then $h+g_{n} \rightarrow h$ weakly in $L^{p}(\mu, X)$ for each 
$1<p<\infty$. Note that, for each $\omega$ in $\Omega$,

$$
\lim _{n \rightarrow \infty}\left\|h(\omega)+g_{n}(\omega)\right\|_{\mathrm{M}}=\|h(\omega)\|_{\mathrm{M}}=1
$$

and $\left\|h(\omega)+g_{n}(\omega)\right\|_{\mathrm{M}} \leq \sqrt{10}\left\|h(\omega)+g_{n}(\omega)\right\|=\frac{1}{2} \sqrt{10}$ for each $n$ in $\mathbb{N}$. Therefore, by the Bounded Convergence Theorem,

$$
\lim _{n \rightarrow \infty}\left\|h+g_{n}\right\|_{L^{p}\left(\mu,\left(X,\|\cdot\|_{M}\right)\right)}=\|h\|_{L^{p}\left(\mu,\left(X,\|\cdot\|_{M}\right)\right)}=1
$$

for each $1<p<\infty$. Thus $L^{p}\left(\mu,\left(X,\|\cdot\|_{\mathrm{M}}\right)\right)$ fails to have $\mathrm{KK}$ even though $\left(X,\|\cdot\|_{\mathrm{M}}\right)$ is MLUR and has $\mathrm{KK}$; the assertion that $\left(X,\|\cdot\|_{\mathrm{M}}\right)$ has KK follows from the fact that $E^{*}$ (and hence $\left(X,\|\cdot\|_{M}\right)$ ) has the Schur property (see [T]). This completes Example 2 with respect to improving the Lin example. But, furthermore, note $X$ has the Radon-Nikodým property because it is a separable dual space. Now, let $p$ with $1<p<\infty$ be fixed. Since $L^{p}\left(\mu,\left(X,\|\cdot\|_{\mathrm{M}}\right)\right)$ fails to have $\mathrm{KK}$, it also fails to have property $(\mathrm{G})$. Hence $\left(X,\|\cdot\|_{\mathrm{M}}\right)$ fails to have property $(\mathrm{G})$; see [L-L2]. Thus (see [D-H-S]) the space $h^{p}\left(D,\left(X,\|\cdot\|_{\mathrm{M}}\right)\right)$ fails to have $\operatorname{KK}(\beta)$. All the listed geometrical properties of this example are summarized in the following concluding result.

Proposition. Let $X$ be the Banach space in Example 2 equipped with the norm $\|\cdot\|_{M}$ and let $(\Omega, \Sigma, \mu)$ be the measure space given there. Then $X$ has the Radon-Nikodym property, has the Schur property (hence has $K K$ ) and is $M L U R$; but $L^{p}(\mu, X)$ fails to have $K K$ and $h^{p}(D, X)$ fails to have $K K(\beta)$ for each $1<p<\infty$.

\section{References}

[A] K.W. Anderson, Midpoint local uniform convexity, and other geometric properties of Banach spaces, Ph.D. Dissertation, University of Illinois, Urbana, Illinois 1960.

[D-H-S] P.N. Dowling, Z. Hu and M.A. Smith, Geometry of spaces of vector-valued harmonic functions, Can. J. Math., 42(2) (1994), 274-283.

[D-U] J. Diestel and J.J. Uhl, Jr., Vector measures, Math Survey 15, Amer. Math. Soc., Providence, RI 1977.

[L] P.K. Lin, unpublished manuscript, 1987.

[L-L1] B-L. Lin and P-K. Lin, Property (H) in Lebesgue-Bochner function spaces, Proc. Amer. Math. Soc., 95 (1985), 581-584.

[L-L2] B-L. Lin and P-K. Lin, Denting points in Bochner $L^{p}$-spaces, Proc. Amer. Math. Soc., 97 (1986), 629-633.

[L-T] J. Lindenstrauss and L. Tzafriri, Classical Banach Spaces I, Ergebnisse der Mathematik und ihrer Grenzgebiete 92, Springer-Verlag, New York 1977.

[M] R.E. Megginson, The semi-Kadec-Klee condition and nearest-point properties of sets in normed linear spaces, Ph.D. Dissertation, University of Illinois, Urbana, Illinois 1983. 
[S] M.A. Smith, A Banach space that is MLUR but not HR, Math. Ann., 256 (1981), 277-279.

[S-T] M.A. Smith and B. Turett, Rotundity in Lebesgue-Bochner function spaces, Trans. Amer. Math. Soc., 257 (1980), 105-118.

[T] M. Talagrand, La propriete de Dunford-Pettis dans $C(K, E)$ et $L^{1}(E)$, Israel J. Math., 44 (1983), 317-321.

Received February 12, 1993 and revised February 22, 1994.

MIAMI UNIVERSITY

OXFORD, OH 45056

E-MAIL ADDRESS: PNDOWLING@MIAVX1.ACS.MUOHIO.EDU

el Paso Community College

El PASO, TX 79998

E-MAIL ADDRESS: DAVIDHU@LAGUNA.EPCC.EDU

AND

Miami University

OXFORD, OH 45056

E-MAIL ADDRESS: MASMITH@MAIMIU.ACS.MUOHIO.EDU 


\section{CONTENTS}

N. Ben Amar, Tangential deformations on the dual of nilpotent special Lie

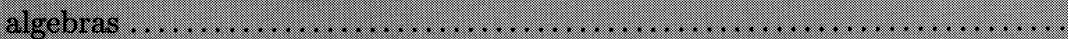

Martin Bendersky, Donald M. Davis and Mark Mahowald, $v_{1}$-periodic

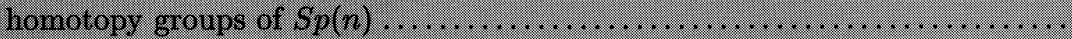

Georgia Benkart, Seok-Jin Kang, Kailash C. Misra, Indefinite Kac-

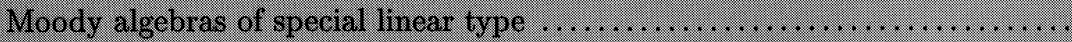

Robin Brooks and Charles Odenthal, Nielsen numbers for roots of maps

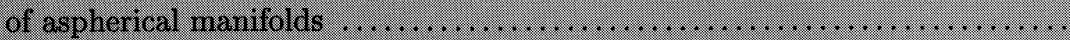

L.J. Bunce and J.D. Maitland Wright, Velocity maps in von Neumann

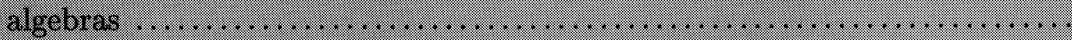

Bradley N. Currey, Smooth decomposition of finite multiplicity monomial representations for a class of completely solvable homogeneous spaces ...... R.J. Daverman and D.F. Snyder, On proper surjections with locally triv-

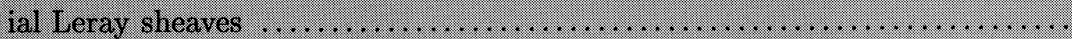

Patrick N. Dowling, Zhibao Hu and Mark A. Smith, MLUR renormings

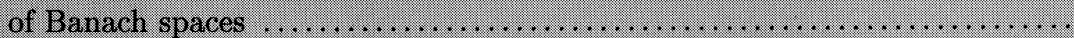
Tuval Foguel, Finite groups with a special 2-generator property ..........

Mourad E.H. Ismail and Mizan Rahman, Some basic bilateral sums and

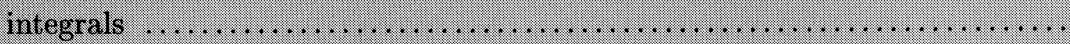

Wojciech Jaworski, Strong approximate transitivity, polynomial growth, and spread out random walks on locally compact groups $\ldots \ldots \ldots \ldots \ldots . . . . . .$.

N. Kutev and F. Tomi, Nonexistence and instability in the exterior Dirichlet problem for the minimal surface equation in the plane............

A. Nobile, Equisingularity Theory for Plane Curves With Embedded

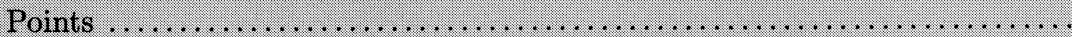

Dominikus Noll, Directional differentiability of the metric projection in Hilbert space 


\section{PACIFIC JOURNAL OF MATHEMATICS}

Volume $170 \quad$ No. $2 \quad$ October 1995

Tangential deformations on the dual of nilpotent special Lie algebras

297

NABIHA BEN AMAR

$v_{1}$-periodic homotopy groups of $S p(n)$

319

MaRTIN BENDERSKY, DONALD M. DAVIS and MARK MAHOWALD

Indefinite Kac-Moody algebras of special linear type

GeOrgia BenKart, SEOK-Jin KANG and KaILASh C. MisRa

Nielsen numbers for roots of maps of aspherical manifolds

405

ROBIN B. S. BROOKS and CHARLES ODENTHAL

Velocity maps in von Neumann algebras

L. J. BUNCE and JOHN DAVID MAITLAND WRIGHT

Smooth decomposition of finite multiplicity monomial representations for a class of 429 completely solvable homogeneous spaces

\section{BRADLEY CURREY}

On proper surjections with locally trivial Leray sheaves

ROBERT JAY DAVERMAN and DAVID FRED SNYDER

MLUR renormings of Banach spaces

PATRICK DOWLING, Zhibao Hu and MARK ANDREW SMIth

Finite groups with a special 2-generator property

TUVAL S. Foguel

Some basic bilateral sums and integrals

MOURAD ISMAIL and MIZAN RAHMAN

Strong approximate transitivity, polynomial growth, and spread out random walks on 517 locally compact groups

WOJCIECH JAWORSKI

Nonexistence and instability in the exterior Dirichlet problem for the minimal surface 535 equation in the plane

NiKolai KuteV and FriedRich TOMI

Equisingularity theory for plane curves with embedded points

Augusto Nobile

Directional differentiability of the metric projection in Hilbert space

DOMINIKUS NOLL 